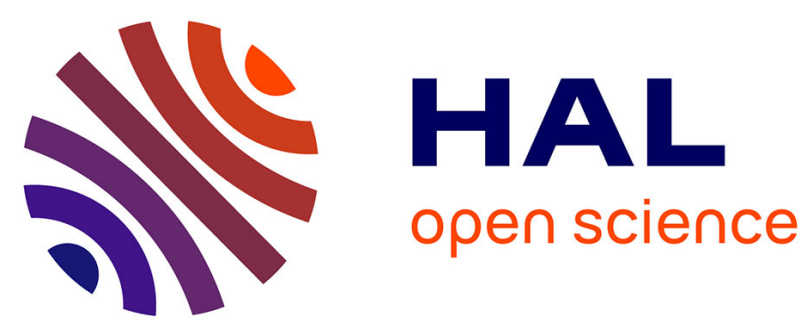

\title{
On the calibration of elastoplastic parameters at the microscale via X-ray microtomography and digital volume correlation for the simulation of ductile damage
}

Ante F Buljac, Victor-Manuel Trejo-Navas, Modesar Shakoor, Amine Bouterf, Jan Neggers, Marc Bernacki, Pierre-Olivier Bouchard, Thilo F. Morgeneyer, François Hild

\section{To cite this version:}

Ante F Buljac, Victor-Manuel Trejo-Navas, Modesar Shakoor, Amine Bouterf, Jan Neggers, et al.. On the calibration of elastoplastic parameters at the microscale via X-ray microtomography and digital volume correlation for the simulation of ductile damage. European Journal of Mechanics - A/Solids, 2018, 72, pp.287-297. 10.1016/j.euromechsol.2018.04.010 . hal-01767392

\section{HAL Id: hal-01767392 \\ https://hal.science/hal-01767392}

Submitted on 16 Apr 2018

HAL is a multi-disciplinary open access archive for the deposit and dissemination of scientific research documents, whether they are published or not. The documents may come from teaching and research institutions in France or abroad, or from public or private research centers.
L'archive ouverte pluridisciplinaire HAL, est destinée au dépôt et à la diffusion de documents scientifiques de niveau recherche, publiés ou non, émanant des établissements d'enseignement et de recherche français ou étrangers, des laboratoires publics ou privés. 


\title{
On the Calibration of Elastoplastic Parameters at the Microscale via X-Ray Microtomography and Digital Volume Correlation for the Simulation of Ductile Damage
}

\author{
Ante Buljac ${ }^{\mathrm{a}, \mathrm{b}}$, Victor-Manuel Trejo Navas ${ }^{\mathrm{c}}$, Modesar Shakoor ${ }^{\mathrm{c}}$, Amine \\ Bouterf $^{\mathrm{a}}$, Jan Neggers ${ }^{\mathrm{a}}$, Marc Bernacki ${ }^{\mathrm{c}}$, Pierre-Olivier Bouchard ${ }^{\mathrm{c}}$, Thilo F. \\ Morgeneyer $^{\mathrm{b}}$, François Hild ${ }^{\mathrm{a}, *}$ \\ ${ }^{a}$ Laboratoire de Mécanique et Technologie (LMT), ENS Paris-Saclay, CNRS, Université \\ Paris-Saclay, 61 avenue du Président Wilson, 94235 Cachan Cedex, France \\ ${ }^{b}$ MINES ParisTech, PSL Research University, Centre des Matériaux, CNRS UMR 7633, \\ BP 87, 91003 Evry, France \\ ${ }^{c}$ MINES ParisTech, PSL Research University, CEMEF-Centre de mise en forme des \\ matériaux, CNRS UMR 7635, CS 10207 rue Claude Daunesse, 06904 Sophia Antipolis \\ Cedex, France
}

\begin{abstract}
An identification framework is introduced herein to calibrate material parameters at the microscale in order to analyze ductile damage. It is applied to study a dog-bone sample, which is made of spheroidal graphite cast iron, loaded in tension and imaged via in situ microtomography. The region of interest is analyzed via Digital Volume Correlation (DVC) to measure kinematic fields. Finite Element (FE) simulations, which account for the studied microstructure that is explicitly meshed thanks its 3D image, are driven by Dirichlet boundary conditions extracted from DVC measurements. The plastic behavior of the ferritic matrix is calibrated via integrated DVC. The three mechanisms of ductile damage are then analyzed in view of the predictions of numerical simulations at the microscopic scale.
\end{abstract}

Keywords: Cast iron; Digital Volume Correlation; elastoplastic material; microstructure calculation; in situ test

\footnotetext{
${ }^{*}$ Corresponding author. Email: hild@lmt.ens-cachan.fr
} 


\section{Introduction}

Motivated by the constant quest for more fuel efficient and hence lighter design substantial progress has been made in understanding and modeling failure of ductile materials over the last decades [1, 2, 3]. Yet, some questions still

5 remain open and robust ductile damage models that would be able to correctly predict fracture loci $\epsilon_{f}(\eta, \mu)$ under arbitrary loading paths are still lacking 4, 5. 6], where $\epsilon_{f}$ denotes the fracture strain, $\eta$ the stress triaxiality and $\mu$ the Lode parameter. One of the main reasons is an incomplete understanding of ductile damage mechanisms at lower scales. The current work is motivated by the idea of having more realistic macroscopic calculations thanks to physical models directly calibrated at the microscale when analyzing two of the three steps of ductile damage, namely, void growth and coalescence. To achieve this aim, the feasibility of an experimental-computational framework allowing for validation and identification of the material properties at the microscale is shown in the following work. To the authors' knowledge, it is the first time that such type of study is reported.

Damage models are useful to optimize the forming processes when dealing with metals and alloys and to predict the in-service life of structures [7, 8]. The first models, which are known as macroscopic postulates, were written at the scale of the volume element in continuum mechanics. They allow damage occurrence and the softening toward fracture to be described [9, 10, 11. However, these models are known to have limited predictive capabilities for complex loading paths and are not easy to calibrate [5].

An alternative to the previous constitutive postulates is to derive the macroscopic response from microscopic formulations [12, 13. The first propositions were based on closed-form derivations [12. Numerical simulations were also proposed to enhance the initial propositions [13]. Over the years, many improvements were proposed to enlarge the validity domain of the developed models [2, 8, 3]. However, it is worth noting that the calibration of such models still 30 is a (very) difficult task [4]. All such simulations were never probed against 
experimental results, in particular the behavior of the matrix, which is usually described by very simple constitutive postulates (e.g., Lukwik's power law). This calls for advanced observation, simulation and identification techniques.

On the experimental side, computed microtomography was used to directly 35 monitor damage in various materials [14, 15, 16, 17]. Physical damage variables such as porosity were observed experimentally thanks to 3D imaging techniques [18, 19, 20, 21. It is possible to study individually inclusions and voids with manual [20] or automatic 21] procedures. The three ductile damage mechanisms, namely, void nucleation [22, 23], growth [24, 25, 26] and coalescence [27, 28, 16, 29] were analyzed and quantified thanks to computed microtomography.

Simulations are needed to experimentally relate observed quantities such as total strain, porosity and the number of fractured/debond inclusions to internal variables (e.g., stresses) to derive criteria for nucleation and coalescence [30].

${ }_{45}$ Averaged damage models were derived by such simulations. The microscale calculations were usually performed with idealized microstructures and constitutive behavior [2, 8, 3]. Moreover, uniform kinematic or static boundary conditions were prescribed. Such conditions cannot capture the local strain and stress states that inclusions and voids are subjected to due to their random shapes and distributions [18, 31, 32, 21, 33]. The effect of the three-dimensional random distribution of voids on softening was also studied 34] using different void volume fractions. It is concluded that it is desirable to work with realistic microstructures.

From the acquired tomographies, real microstructures can be meshed 35 , 55 36. The level set (LS) method [37] is useful to describe interfaces in FE computations when large deformations and complex topological events occur [38, 39, 40]. When FE simulations were run with such interfaces, image processing was directly carried out on FE meshes, thereby making such procedures applicable thanks to parallel implementations [41.

Once such microstructures were generated, the next step was to start validating the numerical simulations. One key aspect is to be able to perform and 
monitor in situ mechanical tests [42, 43. Combined with digital volume correlation, displacement fields can be measured within the bulk of imaged materials. The early developments of DVC [4, 45, 46, 47] consisted of independently registering small interrogation volumes in the considered Region of Interest (ROI). Global approaches [4] perform registrations over the whole ROI by measuring kinematic fields that are, for instance, based on FE discretizations, which assume displacement continuity. Such DVC approaches were directly linked with numerical simulations of mechanical problems [49, 50. They were also used in a numerical framework to partially validate simulations at the microscale [51, 33.

The previously introduced experimental-computational framework [51, 33] is further extended herein to perform the calibration of material parameters at the microscale via 4D mechanical correlation for nodular graphite cast iron. Up to now, such approaches were only performed at the macroscopic level [52]. Once such calibration has been achieved, the next step is to analyze the development of ductile damage. The proposed framework to calibrate material parameters at the microscale is based on the following steps (Figure 1):

1. X-ray microtomography to get $3 \mathrm{D}$ images of an in situ test in a lab tomograph. By post-processing the reconstructed volume in the reference configuration, the morphology of the microstructure is obtained. The interest of using tomography instead of laminography is that the whole sample cross-section can be imaged. Consequently, measured load data will also be used in the present analyses to be compared with predicted resultant forces. Such data were not available in the in situ experiments reported so far on the same material for the analyzed volume of interest [51, 33].

2. Global DVC to measure displacement fields whose kinematic basis is made of the shape functions of 8-noded elements. These displacement fields serve as Dirichlet boundary conditions to FE simulations at the microscale thanks to the interpolation functions of the meshes associated with DVC and FE analyses. It was shown that this step is critical for validation purposes at the microscale [51, 33] and also for identification purposes at 
a mesoscale [52].

3. The FE simulations at the microscale explicitly account for the morphology of the studied material. Therefore the considered meshes made of 4-noded tetrahedra (mixed velocity-pressure formulation with P1+/P1 elements) are adapted to the microstructure by using signed distance functions obtained from the 3D image.

4. FE simulations are run with an elastoplastic constitutive equation to model the nonlinear behavior of the ferritic matrix. The corresponding

100 parameters will be calibrated herein since this is believed to be one cause of discrepancies observed between laminography experiments and simulations [51, 33]. The nodules are assumed to be elastic with very low elastic properties, which is a classical hypothesis when performing damage calculations under tensile loading [53, 54, 55, 56]. This assumption is related to the fact that debonding occurs very early in cast iron. This hypothesis was recently confirmed with DVC measurements [57, 51, 33. More advanced models (e.g., accounting for debonding of the interfaces between nodules) will not be investigated herein.

5. 4D mechanical registration consists of minimizing the registration residuals combined with the global equilibrium gap to calibrate the sought material parameters. This integrated approach allows meshes to be as fine as required as opposed to standard DVC techniques [58, 59, 60]. 


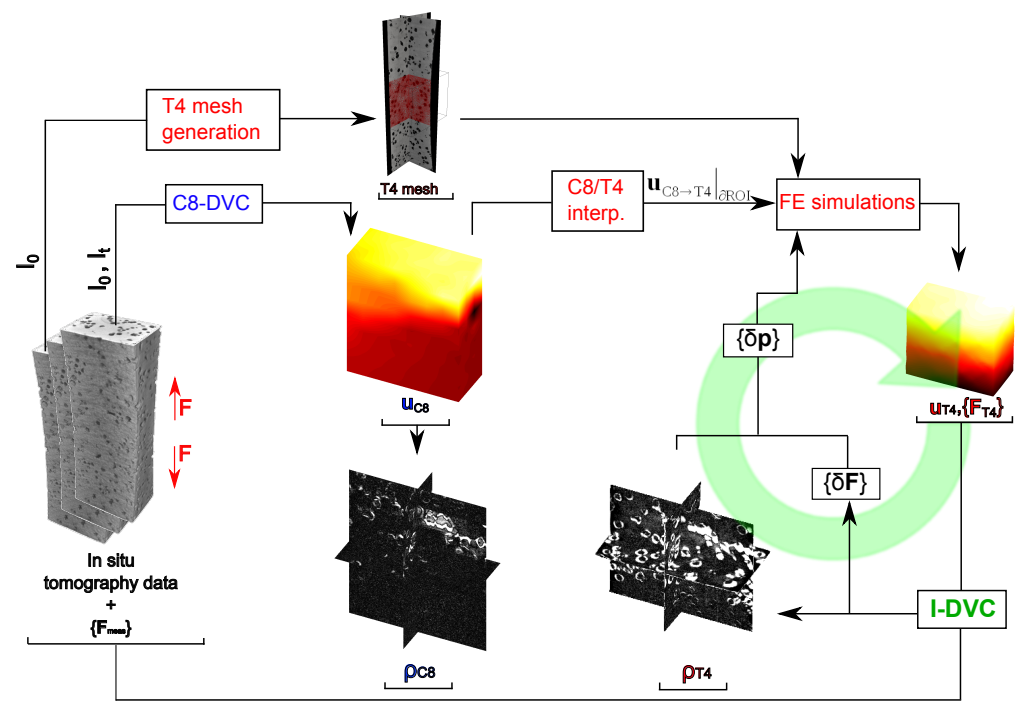

Figure 1: Schematic representation of the methods used in the present paper for calibrating material parameters via numerical simulations at the microscale. Global DVC based on 8noded elements (C8-DVC) is run to register a series of reconstructed volumes $I_{t}$ with respect to the reference configuration $I_{0}$. Such analyses provide the gray level residuals $\rho_{C 8}$, and the measured displacements $\boldsymbol{u}_{C 8}$, which are interpolated on a mesh made of 4-noded tetrahedra (T4). From the reference volume $I_{0}$, a T4 mesh is adapted to the underlying microstructure. Finite element simulations are then run to determine, for any given set of material parameters $\{\boldsymbol{p}\}$, the displacement fields $\boldsymbol{u}_{T 4}$ and the reaction forces $\left\{\boldsymbol{F}_{T 4}\right\}$ to be used in an integrated DVC (I-DVC) approach to compute global equilibrium residuals $\{\delta \boldsymbol{F}\}=\left\{\boldsymbol{F}_{\text {meas }}\right\}-\left\{\boldsymbol{F}_{T 4}\right\}$, and gray level residuals $\rho_{T 4}$

The paper is organized as follows. First the different steps of the identification framework shown in Figure 1 are described in detail. A mesh convergence study is then discussed and finally the damage mechanisms as observed experimentally and predicted numerically are critically assessed and compared.

\section{Identification and validation framework}

\subsection{In situ mechanical experiment}

The material studied herein is commercial nodular graphite cast iron (ENGJS-400 [56]). Figure 2 shows the graphite nodules and a secondary void pop- 
ulation in the matrix as revealed by laminography imaging, subsequent filtering and binarization [61]. The number of contrasted objects per sub-volume (isotropic element length $\ell=70 \mu \mathrm{m}$ ) is of the order of 1 . The volume fraction of nodules is on the order of $11 \%$. The matrix is mostly ferritic.

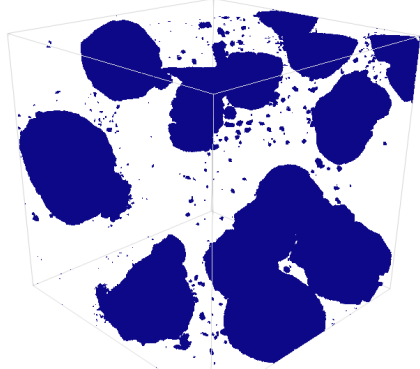

Figure 2: Isometric view of nodules and secondary void population of the reconstructed volume via laminography of the studied cast iron. The region of interest has a size of $280 \times 280 \times 280 \mu^{3}$ (after Ref. 61]

A dog-bone sample was obtained via electro discharge machining (EDM) from a 2-mm thick plate. To ensure that the specimen will break in the ligament area and not in the grips, the central part was thinned with a radius of $20 \mathrm{~mm}$. This zone was 9-mm long, while the smallest cross-section of the sample was $2 \times$ $1 \mathrm{~mm}^{2}$. The tensile test reported herein was carried out in situ within the North Star Imaging X50+ tomograph (Figure 3). The testing machine is similar to one of those designed by Buffière et al. [43. One scan of the sample corresponded to $360^{\circ}$ rotation about its vertical axis. 1,000 radiographs were acquired with a definition of 1,536 $\times 1,944$ pixels. Each scan lasted approximately $1.5 \mathrm{~h}$. The physical voxel length was $3.4 \mu \mathrm{m}$, and the reconstructed volume was encoded with 8-bit deep gray levels. 


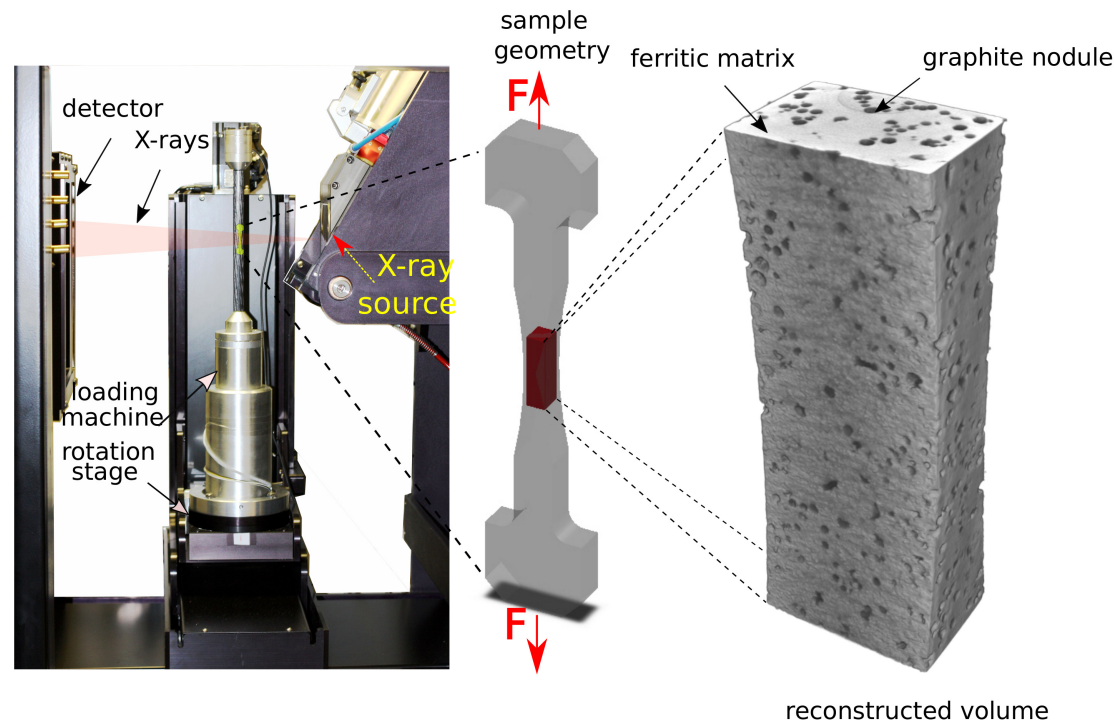

Figure 3: Tomography setup with the representation of the tested sample. The reconstructed region of interest is also shown

The specimen was pulled in six incremental loading and unloading steps (Figure 4) in a custom-made testing machine (Figure 3), which was placed on the rotation stage of the tomograph. The first two scans of the reference configuration allow displacement uncertainties to be evaluated via DVC analyses. Six additional scans were acquired for different applied load levels. When the sample was not unloaded, the scan was started after some dwell time to allow for stress relaxation of the whole tensile stage. The considered load is then equal to the maximum level reached prior to the acquisition process. 


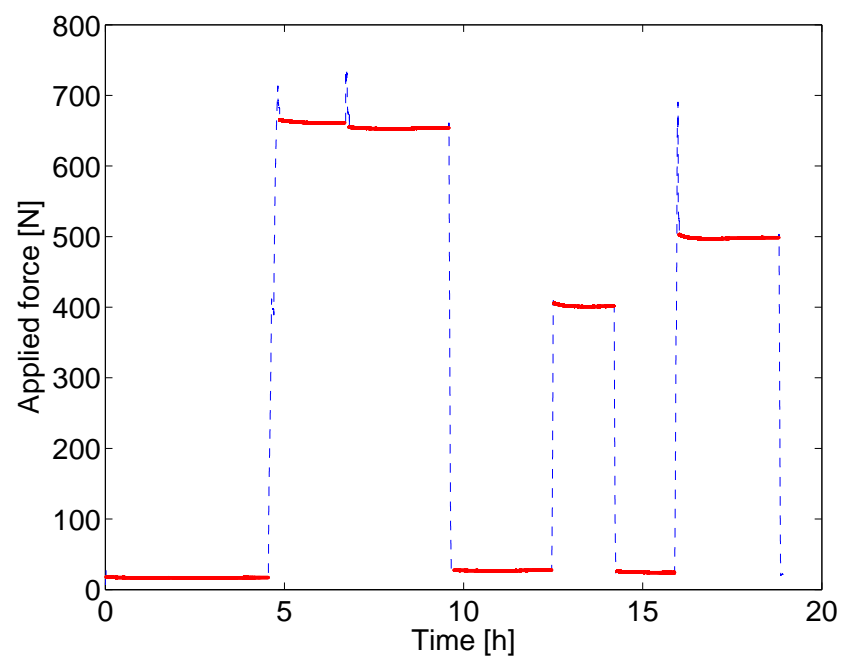

Figure 4: Loading and acquisition histories for the in situ tensile test. The red line shows the tomographic acquisitions. First, two acquisitions were carried out under minimal load for uncertainty quantifications. Then, six additional scans were performed at different load levels for identification purposes

\subsection{Displacement uncertainty}

The reported test is first analyzed via global DVC with meshes made of 8-noded cubes (i.e., it will be referred to as C8-DVC in the sequel). Such measurements allow the experimental boundary conditions to be prescribed to the numerical simulations (Figure 1). It was shown in earlier works on the same type of material that these boundary conditions are crucial when seeking quantitative comparisons with numerical simulations at macroscopic [52] and microscopic [51, 33] scales.

The analyzed ROI is shown in Figure 6(blue mesh). Its size is $320 \times 576 \times 640$ voxels, which corresponds to a volume of $4.64 \mathrm{~mm}^{3}$. Figure 5 shows the change of standard displacement uncertainties as functions of the element length $\ell$ for the three displacement components. The observed trend is to be expected from tomographic data 62 and exemplifies the compromise between measurement uncertainty and spatial resolution [59]. In the following analyses, an element length $\ell=64$ voxels is selected, which leads to a displacement uncertainty of 
the order of 0.12 voxel (i.e., $\approx 0.4 \mu \mathrm{m}$ ). The same order of magnitude (when material for 64-voxel elements [51, 33, 61.

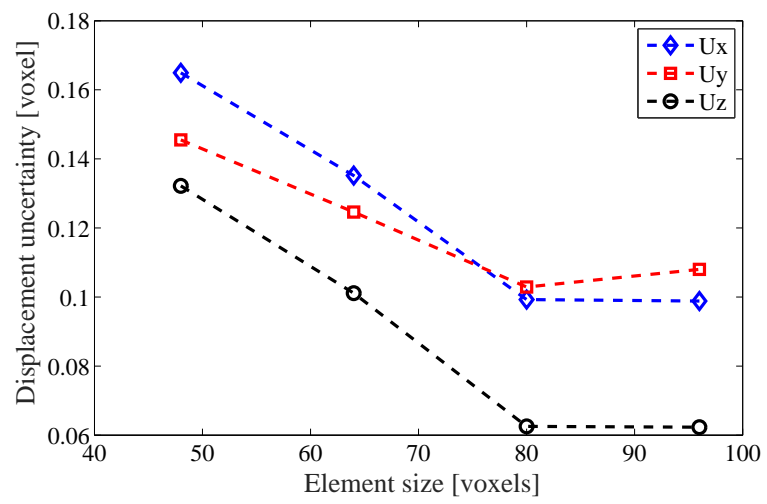

Figure 5: Standard displacement uncertainty as a function of the element length for the three displacement components

\subsection{Meshed microstructure}

In order to model the microstructure observed in the experimental 3D image (Figure 6), a level set based technique is followed. The zero contour of the corresponding signed distance function enables the interfaces to be located. The interested reader is referred to previous work by the authors for details on the mesh generation procedure [33. The meshed ROI in the FE calculations has a total volume of $2.87 \mathrm{~mm}^{3}$, which contains 72.7 million voxels. 


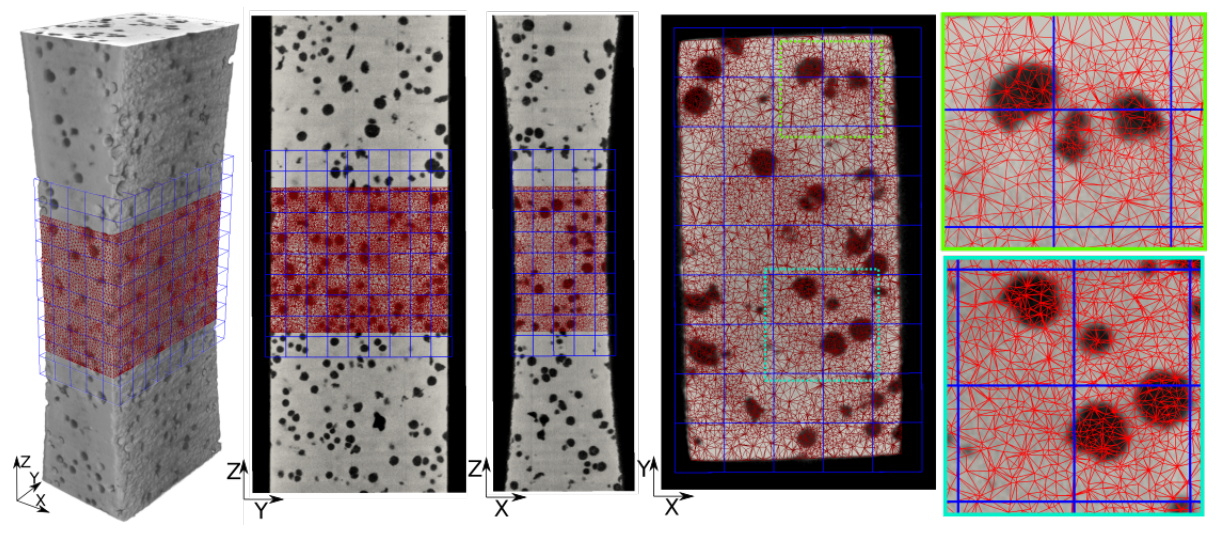

Figure 6: DVC (blue) and FE (red) meshes plotted over the corresponding cast iron microstructure in isometric view and sections normal to $X, Y$ and $Z$ axes. Two regions from the section normal to $Z$ axis are showed enlarged on the right

As explained in Ref. [51, the ROI used for FE simulations has to be included 170 in all DVC domains for any considered loading step. Otherwise displacement fields would not be prescribed on the whole top and bottom boundaries of the FE mesh. In practice, in order to be as representative as possible, a 3D box as large as possible is chosen. Further, to make the following simulations tractable, the meshes should not be made too fine since more than one simulation will have to be carried out to calibrate the plastic parameters of the matrix.

Three different mesh densities have thus been studied to analyze the effect of mesh density on the results reported hereafter (Table 1). The first mesh (Mesh 1), which is very coarse (Figure 7), has less than 13,000 degrees of freedom. The microstructure is only crudely represented for this initial discretization.

Table 1: Structural details of the three studied meshes. The computation time refers to sensitivity fields (for the 3 investigated material parameters) calculations

\begin{tabular}{|c|ccc|}
\hline Mesh & elements & nodes & time (CPUs used) \\
\hline Mesh 1 & 18,785 & 4,293 & $10^{3} \mathrm{~s}(3)$ \\
Mesh 2 & 202,376 & 38,688 & $2 \times 10^{4} \mathrm{~s}(6)$ \\
Mesh 3 & 675,752 & 122,078 & $10^{5} \mathrm{~s}(16)$ \\
\hline
\end{tabular}


Meshes 2 and 3 are finer and describe more faithfully the underlying microstructure (Figure 7). The number of degrees of freedom has been significantly increased to $\approx 110,000$ and $\approx 360,000$ for the finer meshes.

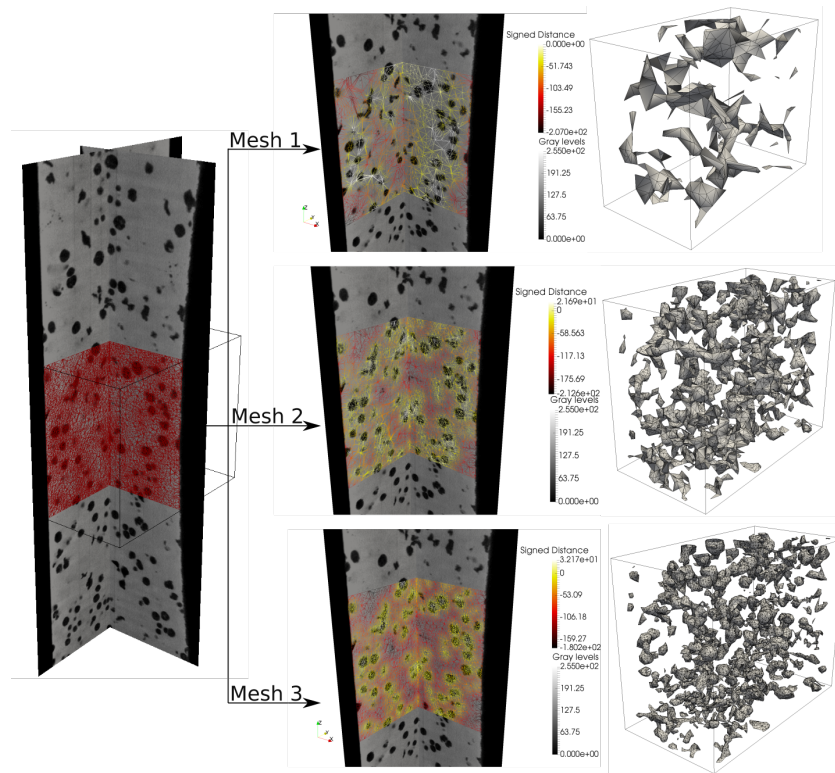

Figure 7: Characterization of three different mesh sizes used in the work with signed distance fields plotted over the corresponding microstructure sections and with the nodule/matrix interface depiction

For FE simulations at these small scales, the mesh quality is of major importance and should be investigated. The quality distribution is reported in Figure 8 for the three mesh densities. A quality of 1 means well shaped tetrahedra, while a quality of 0 means degenerated elements. Finer meshes induce more elements far from interfaces and render mesh adaption easier. Therefore, the histogram for the finest mesh has more elements with good to very good quality. As the meshes are coarsened, their quality is substantially decreased. This result can be understood by analyzing Figure 7. which shows that the 'nodules' correspond to very elongated elements for Mesh 1, which is less the case for Mesh 3. 


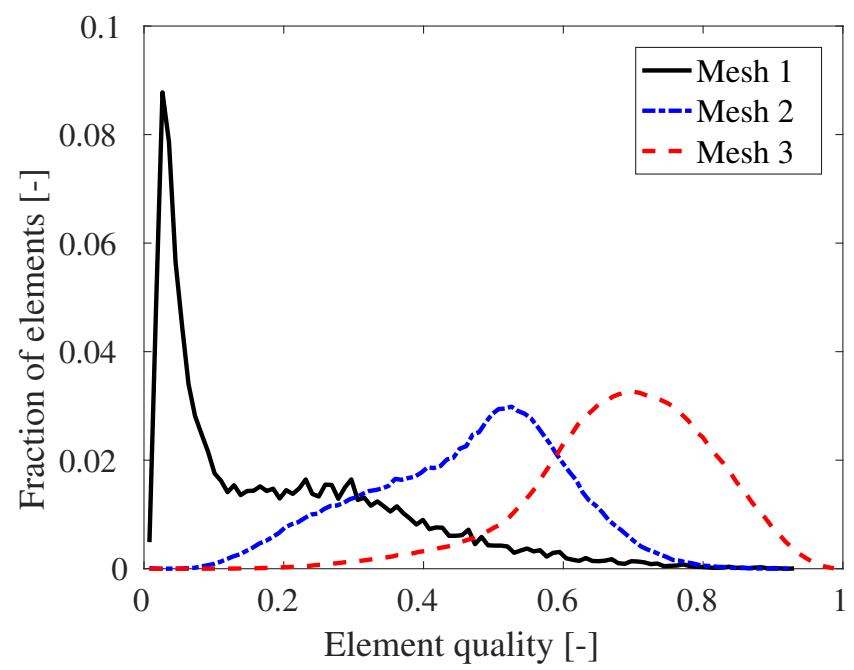

Figure 8: Distribution of mesh quality at the initial state for the three tested meshes

\subsection{Investigated constitutive law}

The framework used for microscale FE simulations is based on previous developments presented in Refs. [39, 63, 41, 64, 65]. To avoid numerical locking in plasticity, a mixed velocity-pressure formulation is used with $\mathrm{P} 1+/ \mathrm{P} 1$ elements [66]. Large transformations are considered with an updated Lagrangian formulation, which is commonly used in metal forming simulations 67. This incremental formulation has proven to give accurate results for small time steps.

The constitutive equation investigated herein is Ludwik's law 68, which describes isotropic hardening as follows

$$
\sigma_{e q}=\sigma_{y}+K p^{n}
$$

where $\sigma_{e q}$ is the second (von Mises) invariant of the deviatoric part of the Cauchy stress tensor, $p$ the accumulated plastic strain associated with the Cauchy strain tensor, $\sigma_{y}$ the yield stress, $K$ the hardening modulus and $n$ the hardening exponent. Such type of constitutive equation may seem very crude for the matrix. This is certainly the case up to an extent that will be investigated herein. ${ }_{205}$ However, almost all cell calculations [12, 13, 2, 3] or even with more complex microstructures [34, 41, 51] use von Mises plasticity. Consequently, the present 
work aims at calibrating material parameters to be as consistent as possible to experimental observations. It is worth noting that the proposed framework is compatible with more advanced constitutive postulates. However, the computa-

desirable thanks to the strong coupling between experiment and simulation [52].

When performing integrated DVC the displacement fields are mechanically admissible (i.e., they satisfy equilibrium in the finite element sense for a chosen constitutive law in addition to their kinematic admissibility thanks to the use of measured boundary conditions). The first 3D developments were based on elastic simulations for which a non-intrusive framework was implemented [60]. 
They were then extended to nonlinear analyses at the macroscale [52]. The FE code was then used to generate kinematic fields that are needed for DVC purposes. When material parameters are sought, the corresponding spatiotemporal sensitivities [69, 70] are also needed for nonlinear constitutive postulates. The $4 \mathrm{D}$ kinematics is thus parameterized with the sought corrections $\{\delta \mathbf{p}\}$ to the current estimate of material parameters $\{\widehat{\mathbf{p}}\}$

$$
\mathbf{u}(\mathbf{x}, t,\{\mathbf{p}\})=\mathbf{u}(\mathbf{x}, t,\{\widehat{\mathbf{p}}\})+\left[\frac{\partial \mathbf{u}}{\partial\{\mathbf{p}\}}(\mathbf{x}, t,\{\widehat{\mathbf{p}}\})\right]^{\dagger}\{\delta \mathbf{p}\}
$$

where $[\partial \mathbf{u} / \partial\{\mathbf{p}\}]$ is the matrix gathering all the spatiotemporal sensitivity fields to the sought material parameters, $\mathbf{x}$ any voxel within the analyzed region of interest, and $t$ time. Thus the discretized displacement field becomes

$$
\mathbf{u}(\mathbf{x}, t,\{\mathbf{p}\})=\sum_{t} \sum_{n} v_{n}(\{\mathbf{p}\}, t) \mathbf{N}_{n}(\mathbf{x})
$$

in which the kinematic degrees of freedom $v_{n}$, which are associated with the shape function vector $\mathbf{N}_{n}$, are not independent but all linked via their sensitivities to the sought parameters

$$
v_{n}(\{\mathbf{p}\}, t)=v_{n}(\{\widehat{\mathbf{p}}\}, t)+\left\{\frac{\partial v_{n}}{\partial\{\mathbf{p}\}}(\{\widehat{\mathbf{p}}\}, t)\right\}^{\dagger}\{\delta \mathbf{p}\}
$$

The kinematic sensitivities associated with the chosen spatial discretization are gathered in matrix $\left[\mathbf{S}_{\boldsymbol{v}}(t)\right]$ (i.e., $\left.\left(S_{v}(t)\right)_{n m}=\left(\partial v_{n} / \partial p_{m}\right)(t)\right)$ that is evaluated for each time $t$. The finite difference method is used to approximate $\frac{\partial v_{n}}{\partial\{\mathbf{p}\}}$

$$
\frac{\partial v_{n}}{\partial\{\mathbf{p}\}}(\{\widehat{\mathbf{p}}\}, t) \approx \frac{v_{n}(\{\widehat{\mathbf{p}}\}+\zeta\{\widehat{\mathbf{p}}\}, t)-v_{n}(\{\widehat{\mathbf{p}}\}, t)}{\zeta \widehat{\mathbf{p}}}
$$

where $\zeta$ is a perturbation factor. To illustrate such fields, Figure 9 shows the longitudinal component of the sensitivity fields associated with the three plastic parameters when each of them is independently perturbed by $\zeta=2 \%$ with 235 respect to its nominal value. Consequently, these fields are expressed in voxels divided by the physical unit of the studied parameters. It is observed that the first and last loading steps have overall shapes that are different from the intermediate steps (i.e., 2 to 5). This trend may signal the development of different damage mechanisms or regimes, as will be discussed in Section 3.2 . 


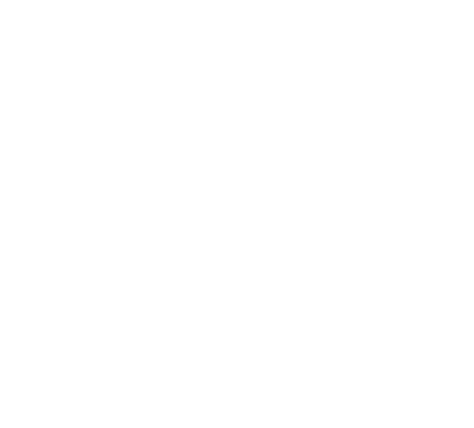

(a) Yield stress (voxel/MPa)

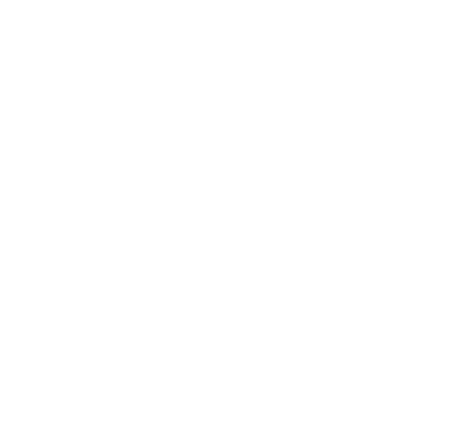

(b) Hardening modulus (voxel/MPa)

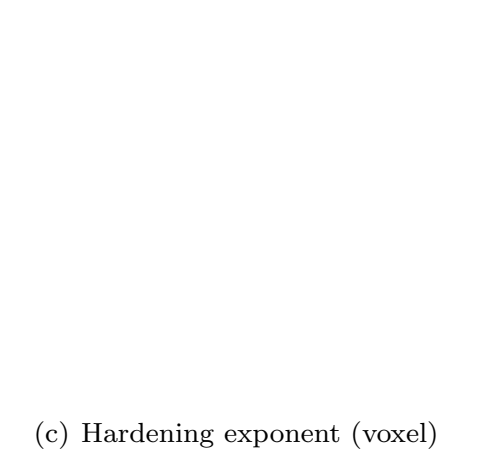

Figure 9: Sensitivity fields in the longitudinal direction for the last loading step. Online version: corresponding movie for the 6 analyzed steps. The color bar has a fixed range

Table 1 reports the computation time required to have access to sensitivity fields for the three investigated material parameters (i.e., four FE simulations). There is a two order of magnitude difference in computation time. It is worth noting that the machines used to run these different calculations had to be adapted to the mesh density, namely, for Meshes 1 and 2, a workstation (6 CPUs) with Intel(R) Xeon(R) E5 processor (32 GB, $3.3 \mathrm{GHz})$ was used, and a node of a cluster (16 CPUs) with $\operatorname{Intel}(\mathrm{R}) \mathrm{Xeon}(\mathrm{R}) \mathrm{E}$ 7-8837 processor (252 $\mathrm{GB}, 2.67 \mathrm{GHz}$ ) for Mesh 3. For the finest mesh, each set of sensitivity fields (for three material parameters) required about $28 \mathrm{~h}$ of simulation.

$4 \mathrm{D}$ mechanical correlation consists of minimizing the sum of squared gray 
level differences directly with respect to $\{\mathbf{p}\}$

$$
\{\mathbf{p}\}_{\text {meas }}=\underset{\{\mathbf{p}\}}{\operatorname{argmin}} \sum_{\left[0, t_{\max }\right]} \sum_{R O I} \frac{\left(I_{0}(\mathbf{x})-I_{t}(\mathbf{x}+\mathbf{u}(\mathbf{x}, t,\{\mathbf{p}\}))\right)^{2}}{2 \gamma_{I}^{2}}
$$

over the considered ROI for the reconstructed volumes in the reference configuration $I_{0}(\mathbf{x})$ and deformed configuration $I_{t}(\mathbf{x})$. The coefficient $\gamma_{I}^{2}$ denotes the variance associated with acquisition and reconstruction noise of the gray level volumes.

In the present case, load measurements gathered in vector $\left\{\mathbf{F}_{\text {meas }}\right\}$ are also available. Thus they are compared with the FE predictions in which the reaction force vector $\left\{\mathbf{F}_{F E}\right\}$ is due to the fact that measured displacements are prescribed on the top and bottom boundaries of the considered ROI. The global equilibrium gap over the whole loading history reads

$$
\rho_{F}^{2}=\frac{1}{\gamma_{F}^{2}}\left\|\left\{\mathbf{F}_{\text {meas }}-\mathbf{F}_{\text {meas }}(\{\mathbf{p}\})\right\}\right\|^{2}
$$

where $\gamma_{F}^{2}$ denotes the variance of the measurement uncertainty of load cells. The latter is assumed to be white and Gaussian. The minimization of $\rho_{F}^{2}$ alone corresponds to updating the FE model by considering only global equilibrium to estimate the sought parameters $\{\mathbf{p}\}$. It is referred to as load-based FE model updating (or FEMU-F [71, 72]).

In the following, both sets of data, namely, reconstructed volumes and measured loads will be considered in a single approach. A normalization framework is considered [52] in which each considered information is weighted by its merit (i.e., variance and covariance with all the other data) for DVC (i.e., $\bar{\chi}_{I}^{2}$ ) and global equilibrium (i.e., $\bar{\chi}_{F}^{2}$ ). An equal weighting is then assumed between the kinematic and load contributions

$$
\bar{\chi}^{2}=\frac{1}{2} \bar{\chi}_{I}^{2}+\frac{1}{2} \bar{\chi}_{F}^{2}
$$

with

$$
\begin{aligned}
\bar{\chi}_{I}^{2} & =\frac{1}{n_{t}} \sum_{\left[0, t_{\max }\right]} \chi_{I}^{2}(t)=\frac{1}{|R O I| n_{t}} \sum_{\left[0, t_{\max }\right]} \sum_{R O I} \frac{\left(I_{0}(\mathbf{x})-I_{t}(\mathbf{x}+\mathbf{u}(\mathbf{x}, t,\{\mathbf{p}\}))\right)^{2}}{2 \gamma_{I}^{2}} \\
\bar{\chi}_{F}^{2} & =\frac{1}{n_{t}} \chi_{F}^{2}(t)=\frac{1}{n_{F} n_{t}} \sum_{t} \frac{\left\|\left\{\mathbf{F}_{\text {meas }}(t)-\mathbf{F}_{E F}(t,\{\mathbf{p}\})\right\}\right\|^{2}}{\gamma_{F}^{2}}
\end{aligned}
$$


where $n_{F}$ denotes the number of load measurements per scan (i.e., $n_{F}=1$ in the present case). With the chosen weighting, when the residuals are close to unity, the only contribution to the error is due to noise associated with tomography and load cell(s) measurements. If $\bar{\chi}_{I}$ and/or $\bar{\chi}_{F}$ are significantly larger than unity then there are numerical model errors.

$4 \mathrm{D}$ mechanical correlation thus consists of minimizing $\bar{\chi}^{2}$ with respect to the sought parameters

$$
\{\mathbf{p}\}_{\text {meas }}=\underset{\{\mathbf{p}\}}{\operatorname{argmin}} \bar{\chi}^{2}
$$

A Gauss-Newton scheme is implemented for which the corrections $\{\delta \mathbf{p}\}$ to the sought material parameters are iteratively updated [52]. Each iteration lasts about one day. To speed-up the simulations, the sensitivity fields are no longer updated when all parameter variations are less than $10 \%$ of their current estimate. Convergence is reached when their relative variations are all less than $0.01 \%$. uses in a non-intrusive way FE codes to compute the spatiotemporal sensitivity fields in addition to the current estimates of the displacement fields and reaction forces. The inputs to such simulations are the measured displacements of the top and bottom boundaries of the ROI. In the present case, the CimLib library 275 is used with its built-in constitutive laws 63.

\section{Simulation results}

\subsection{Mesh sensitivity}

Table 2 gathers the parameters at the end of the integrated DVC analyses for the three different meshes. The first set of simulations is initialized with the parameters proposed by Zhang et al. 54. The second set of simulations is performed when the previous initial guess is perturbed by $10 \%$. Each set of calibrated parameters is different. 
Table 2: Plastic properties of nodular cast iron obtained via I-DVC

\begin{tabular}{|c|cc|ccc|}
\hline Method & $E(\mathrm{GPa})$ & $\nu$ & $\sigma_{y}(\mathrm{MPa})$ & $K(\mathrm{MPa})$ & $n$ \\
\hline Initial parameters [54 & 210 & 0.3 & 290 & 382 & 0.35 \\
\hline I-DVC, Mesh 1 & 210 & 0.3 & 232 & 303 & 0.23 \\
I-DVC, Mesh 2 & 210 & 0.3 & 235 & 313 & 0.19 \\
I-DVC, Mesh 3 & 210 & 0.3 & 245 & 330 & 0.21 \\
\hline Initial parameters (perturbed) & 210 & 0.3 & 260 & 420 & 0.31 \\
\hline I-DVC, Mesh 1 & 210 & 0.3 & 206 & 349 & 0.22 \\
I-DVC, Mesh 2 & 210 & 0.3 & 211 & 364 & 0.21 \\
I-DVC, Mesh 3 & 210 & 0.3 & 219 & 371 & 0.23 \\
\hline
\end{tabular}

To better understand the difference of all these parameter sets, Figure 10 illustrates the corresponding equivalent stress / strain responses. In comparison with the initial guesses, there are two groups of results. First the two finer mesh densities lead to very similar stress / strain responses. Conversely, the coarser mesh leads to significantly different responses. This difference is attributed to the fact that Mesh 1 only crudely describes the underlying microstructure (Figure 7). The fact that Meshes 2 and 3 yield very similar stress / strain 290 responses indicates that convergence is achieved in terms of matrix behavior accounting for void growth. 


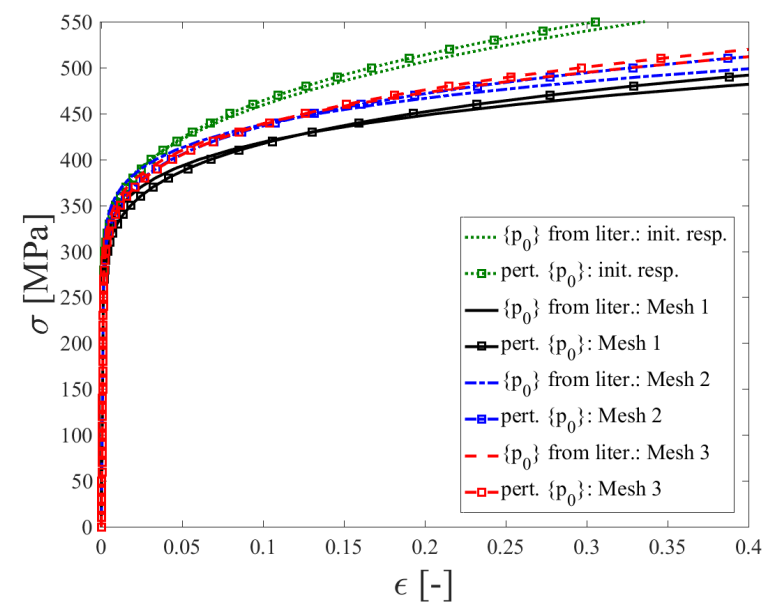

Figure 10: $\quad$ Stress-strain for the cases analyzed in Table 2 In the legend $\left\{\mathbf{p}_{0}\right\}$ and perturbed $\left\{\mathbf{p}_{0}\right\}$ stand for different sets of initial parameters detailed in Table 2 Initial response stands for $\sigma-\epsilon$ values obtained with the initial set of parameters

Since both initial guesses lead to virtually identical stress-strain responses for the three mesh densities (Figure 10), only the initialization based on literature data is further discussed. In the present case, it can be noted that there is a clear difference between the initial guess and the converged solution. This is to be expected since the in situ properties of the present ferritic matrix are not necessarily identical to the ferrite studied by Zhang et al. 54. However, the initial part of the yielding regime (i.e., up to $5 \%$ equivalent plastic strain) is very close. Conversely, for equivalent strains greater than $10 \%$ the difference is more pronounced. It is believed that this is due to the fact that the constitutive law is probed over a larger strain range in the present case thanks to measurements at the microscopic scale.

The local stress-strain differences (Figure 10) will also induce different dimensionless load residuals (see Figure 11). There is a significant decrease of the latter ones from the initial guess to the converged solution. The coarse mesh leads to a higher global residual (i.e., $\bar{\chi}_{F}=14.6$ ) in comparison with the two fine meshes, which lead to global load residuals 9 times the standard uncertainty 
of the load cell.

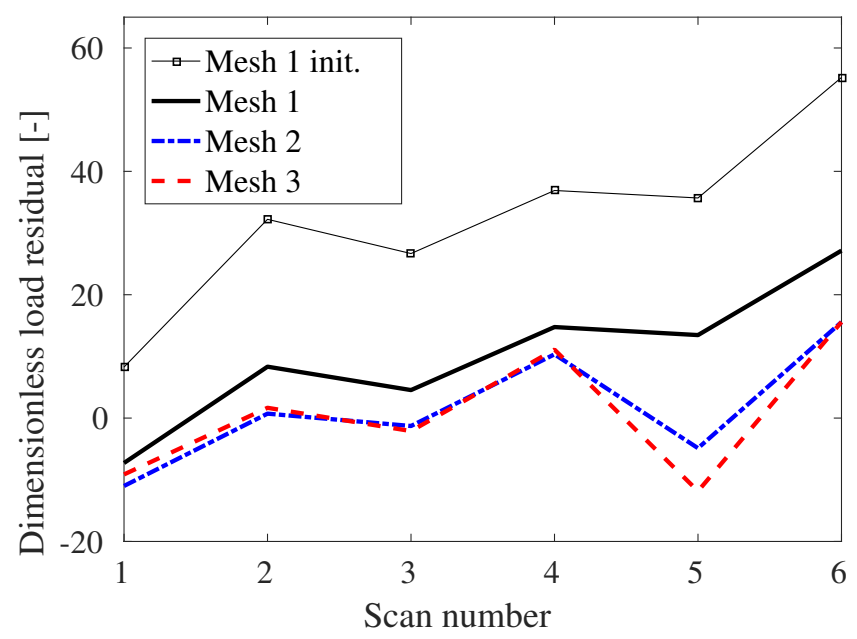

Figure 11: Initial and final dimensionless static residual $\chi_{F}$ for the different mesh sizes used in integrated DVC

Let us recall that the gray level residual $\chi_{I}(t)$ corresponds to the root mean difference between undeformed and corrected deformed volumes by the measured (DVC) or the simulated (FE) kinematic fields, see Equation (9). Figure 12 shows the change of dimensionless gray level residual for the six analyzed loading steps. Even though the C8-DVC analyses are only based on a very coarse mesh (Figure 6) they still lead to the lowest gray level residuals. The latter ones are at least equal to 1.6 times those corresponding to the uncertainty analysis discussed in Section 2.2 This trend indicates that the measured kinematics does not completely follow the details of the material microstructure. However, the residuals remain sufficiently low to deem the measurements trustworthy and apply them as Dirichlet boundary conditions to the FE simulations. 


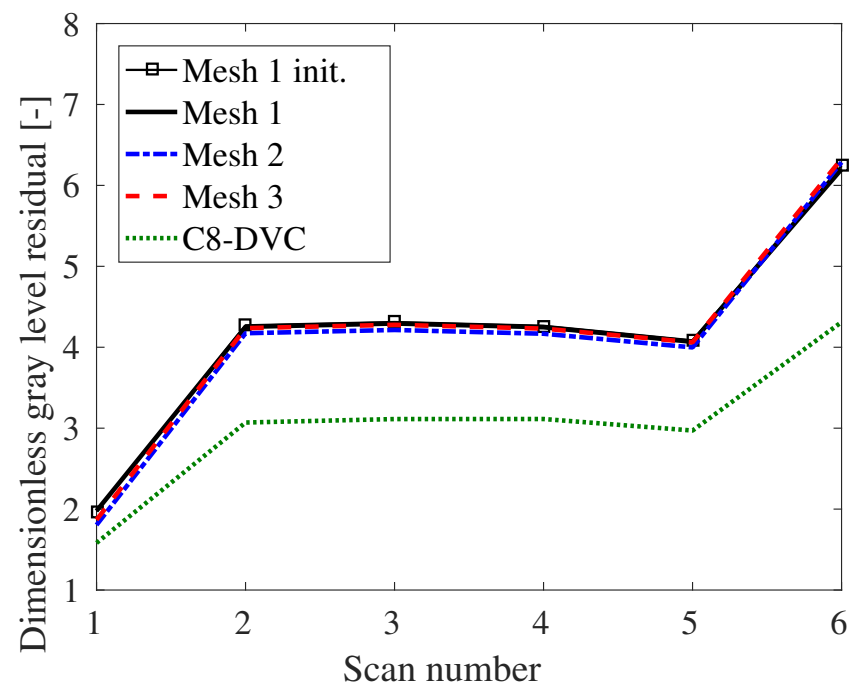

Figure 12: Initial and final dimensionless gray level residuals $\chi_{I}$ for different mesh sizes used in the I-DVC procedure and for DVC calculations

The same degradation of the gray level residuals is observed for the four analyzed numerical cases. Since measured boundary conditions are considered, gray level residuals can also be evaluated for displacement fields that would not be measurable with a standard DVC code since the mesh size would be too small to ensure good convergence. In the present case, all I-DVC results lead to virtually the same residuals. The reason why there is only a very minor influence of the three discretization is due to the fact that the modeled region of interest is small and that the type of loading is rather simple (i.e., induced by uniaxial tension). Further, they are significantly higher than C8-DVC residuals. This clearly indicates a model error, which has to be analyzed more locally by using the voxel scale representation of the gray level residuals (see Section 3.2).

To conclude the mesh sensitivity analysis of the three mesh densities considered herein, the coarser Mesh 1 is disqualified since it is not sufficiently close to the actual microstructure (Figure 7) and it does not predict the overall load level as accurately as the other two meshes. The latter ones lead to very similar load residuals (Figure 11) and stress / strain responses (Figure 10). Consequently, 
they are equivalent in overall predictive power with the chosen constitutive framework. However, in terms of overall computation time there is a significant increase, which was anticipated from the computation of the sensitivity fields (Table 1), namely, $50 \mathrm{~h}$ for Mesh 2 and $236 \mathrm{~h}$ for Mesh 3. It is worth noting that the identification procedure can start with Mesh 1 for the first parameter estimations and finish with Mesh 3 for more detailed analyses.

\subsection{Discussion}

Since the actual microstructure of the material is explicitly meshed, void growth should be reasonably described by the simulations, provided the constitutive law of the matrix is representative of the actual material. Conversely, damage nucleation and coalescence are not accounted for if coalescence mechanisms other than internal necking between nodules are at play. Therefore it is believed that some of the deviations of the previous residuals can be linked to these hypotheses.

Figure 13 shows sections of the gray level residuals for the six (in printed version only sixth) analyzed loading step(s). For the first loading step, the C8DVC residuals reveal that early debonding occurs in particular in the lower/left region of the analyzed volume. For the second loading step, coalescence and fracture has already started and matrix/nodule debonding has further developed over a large area. For I-DVC, the chosen constitutive law is not able to fully describe the response of the material in the transverse directions. Consequently the gray level residuals are higher than in C8-DVC (see Figure 12). Similar observations can be made by analyzing the residuals for steps 3 to 5 , which have essentially the same features. Last, further coalescence and fracture develop in

the last loading step and the residuals degrade for C8-DVC and I-DVC. 


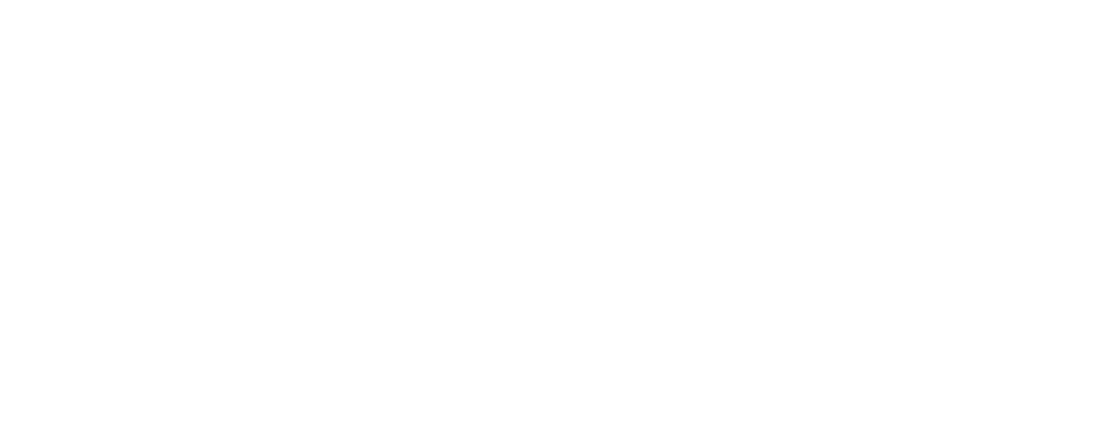

Figure 13: Gray level residuals for I-DVC (left) and C8-DVC (right) analyses for the last loading step. Online version: corresponding movie for the 6 analyzed steps

It is worth remembering that as the analyzed loading step increases, so does the load residual (Figure 11). With the present observations it can be stated that this degradation is linked to coalescence and fracture. Let us also note that apart from matrix/nodule debonding and coalescence, no other zone with high residuals is observed. Consequently, it is believed that damage nucleation, if present, occurs at scales lower than that of the voxels (i.e., $3.4 \mu \mathrm{m}$ ).

Figure 14 shows microstructural and equivalent strain maps (from FE calculations) for the six analyzed loading steps (in printed version for the sixth loading step). From the first loading step on, the strain field is heterogeneous but the most loaded zone is not the one that will lead to coalescence and fracture. For the second loading step, the zone where coalescence occurs is the most strained but coalescence and more importantly fracture are not captured in FE simulations. Furthermore, there is a second zone where strains concentrate (see loading steps 2 to 5 ). For the last loading step, the strain levels continue to increase.

The FE framework used herein naturally takes into account coalescence by internal necking without any special criterion. In fact, Figure 14 (strain maps, between increments 1 and 4) shows that coalescence instances at about one quarter of the length of the crack are captured in the FE simulation. Hence, if coalescence occurs by internal necking, it should be predictable. If coalescence is not completely captured by FE simulations as in the present case, the individual 
void growth is probably underestimated in addition to nucleation on secondary particles.
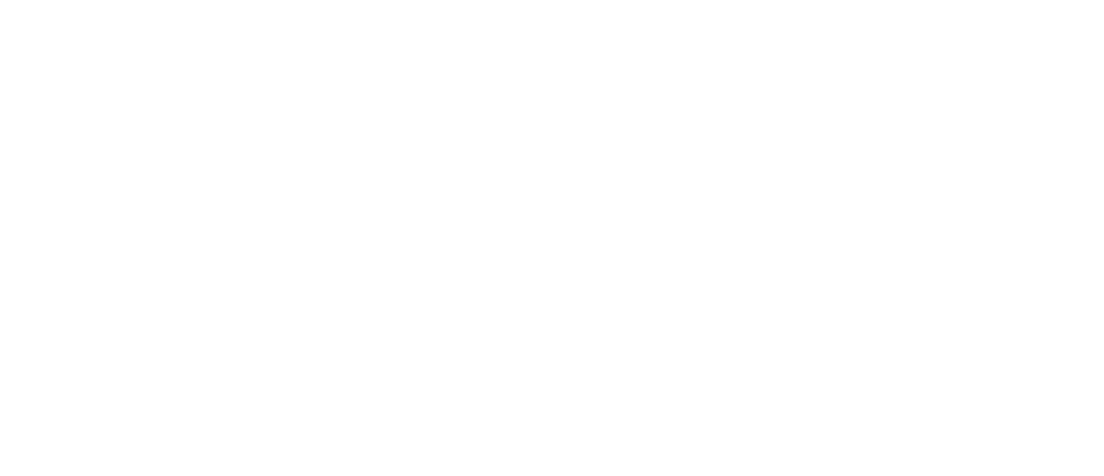

Figure 14: Microstructure section and equivalent strain field (from FE calculations) plotted on the deformed configuration for the last loading step. Online version: corresponding movie for the 6 analyzed steps

The possible reasons for underestimating void growth originating from the matrix behavior are:

- not considering the effects of softening due to damage at smaller scales. The possible acceleration of void growth when coalescence arises calls for an implementation of a coupled damage model that was not considered herein,

- the present hardening law does not saturate (i.e., there is no plateau) and induces unrealistic hardening. This effect will suppress levels of plastic strain in the inter-void ligaments for the high stress states (i.e., postpone void growth and coalescence),

- crystallographic effects of the matrix.

Another reason would be having underestimated the initial void volume fraction in the FE simulation. The quality of the geometrical descriptions of the nodules is still imperfect due to the limited mesh density (even Mesh 3). In this scenario, an underestimated initial void volume fraction in the FE ROI will generally decrease the void growth rate in the matrix. Also, the topological details 

and void interaction in the inter-void ligaments) are not properly captured.

\section{Conclusions}

In this paper the feasibility of an innovative identification procedure (Figure 11 was proven to calibrate matrix parameters for the simulation of damage growth and coalescence at the microscale. Experimental data (i.e., reconstructed volumes) were collected via lab tomography while performing an in situ tensile test on a dog-bone specimen made of nodular cast iron. Bulk kinematic fields in the central region were measured via DVC. The FE mesh is adapted to the real microstructure with a (re)meshing procedure based on level set fields.

The subsequent FE simulations are driven by Dirichlet boundary conditions extracted from DVC measurements. To be as representative as possible of the actual test, the plastic parameters of the ferritic matrix are calibrated utilizing integrated DVC. Graphite nodules are assumed to behave as voids.

The fact that FE simulations were driven by measured boundary conditions allowed the simulations to be more representative of the actual experiment compared to other methods used for obtaining the boundary conditions [33]. It also enabled calibration procedures to be run at the microscale by accounting for load and imaged microstructure since the whole sample cross-section was imaged. Consequently, the constitutive law of the matrix was not only probed in terms of overall load level but also by kinematic fields induced by the random microstructure.

The overall residual levels were sufficiently low, thereby indicating successful identification. The areas of higher residuals corresponded to the position of the debond zones that appear early on, and increased further due to void coalescence. The fact that the numerical simulations did not account for accelerated void growth (i.e., softening at smaller scales) in the coalescence zone explained why the gray level residuals degraded between DVC and I-DVC. Even though more advanced constitutive models may be considered in the future, the overall 
dimensionless residual was only on the order of 8 , which is low in comparison to analyses at the macroscale [52].

One of the next steps of the present work will deal with void sheeting coalescence that was not accounted for in the reported results. Different criteria may then be probed. Similarly, the hypothesis of considering nodules as voids can also be relaxed and debonding may be addressed. The proposed experimentalnumerical framework can still be used to deal with these different degradation mechanisms. On the experimental side, more scans would be needed in particular at earlier loading steps to better capture the matrix behavior prior to significant damage growth.

\section{Acknowledgements}

The financial support of Agence Nationale de la Recherche (ANR-14-CE070034-02 COMINSIDE and ANR-10-EQPX-37 MATMECA grants) is gratefully acknowledged. It is also a pleasure to acknowledge the support of BPI France ("DICCIT" project), and of the Carnot M.I.N.E.S institute ("CORTEX" project). M. Kuna, L. Zybell and M. Horn from IMFD, TU Freiberg, are thanked for material supply as well as for scientific discussions.

\section{References}

[1] J. Besson, Continuum models of ductile fracture: A review, Int. J. Damage Mech. 19 (2010) 3-52.

[2] A. Benzerga, J. Leblond, Ductile fracture by void growth to coalescence, Adv. Appl. Mech. 44 (2010) 169-305.

[3] A. Pineau, A. Benzerga, T. Pardoen, Failure of metals I: Brittle and ductile fracture, Acta Mat. 107 (2016) 424-483.

[4] T.-S. Cao, E. Maire, C. Verdu, C. Bobadilla, P. Lasne, P. Montmitonnet, P.-O. Bouchard, Characterization of ductile damage for a high carbon steel 
using 3D X-ray micro-tomography and mechanical tests - Application to the identification of a shear modified GTN model, Comput. Mat. Sci. 84 (2014) 175-187.

[5] T.-S. Cao, C. Bobadilla, P. Montmitonnet, P.-O. Bouchard, A comparative study of three ductile damage approaches for fracture prediction in cold forming processes, J. Mat. Proc. Technol. 216 (2015) 385-404.

[6] N. Thomas, S. Basu, A. Benzerga, On fracture loci of ductile materials under non-proportional loading, Int. J. Mech. Sci. 117 (2016) 135-151.

[7] J. Lemaitre, R. Desmorat, Engineering Damage Mechanics, Springer, Berlin (Germany), 2005.

[8] G. Z. Voyiadjis (Ed.), Handbook of Damage Mechanics, Springer, New York, NY (USA), 2014.

[9] L. Kachanov, Time of the rupture process under creep conditions, Bull. SSR Acad. Sci., Division of Technical Sciences (in Russian) 8 (1958) 26-31.

[10] Y. Rabotnov, On the Equations of State for Creep, McMillan, New York 470 (USA), 1963, pp. 307-315.

[11] J. Lemaitre, A Course on Damage Mechanics, Springer-Verlag, Berlin (Germany), 1992.

[12] A. Gurson, Continuum Theory of Ductile Rupture by Void Nucleation and Growth: Part I - Yield Criterion and Flow Rules for Porous Ductile Media, ASME J. Eng. Mat. Techn. 99 (1977) 2-15.

[13] A. Needleman, V. Tvergaard, An analysis of ductile rupture in notched bars, J. Mech. Phys. Solids 32 (6) (1984) 461-490.

[14] J. Baruchel, J. Buffière, E. Maire, P. Merle, G. Peix (Eds.), X-Ray Tomography in Material Sciences, Hermès Science, Paris (France), 2000. 
[15] E. Maire, A. Fazekas, L. Salvo, R. Dendievel, S. Youssef, P. Cloetens, J. Letang, X-ray tomography applied to the characterization of cellular materials. related finite element modeling problems, Compos. Sci. Tech. 63 (16) (2003) 2431-2443.

[16] L. Salvo, P. Cloetens, E. Maire, S. Zabler, J. J. Blandin, J. Y. Buffiere,

[20] T. Ueda, L. Helfen, T. F. Morgeneyer, In situ laminography study of threedimensional individual void shape evolution at crack initiation and comparison with Gurson-Tvergaard-Needleman-type simulations, Acta Mat. 78 (2014) 254-270.

[21] F. Hannard, T. Pardoen, E. Maire, C. Le Bourlot, R. Mokso, A. Simar, Characterization and micromechanical modelling of microstructural heterogeneity effects on ductile fracture of 6xxx aluminium alloys, Acta Mat. 103 (2016) 558-572.

[22] C. Landron, O. Bouaziz, E. Maire, J. Adrien, Characterization and modeling of void nucleation by interface decohesion in dual phase steels, Scripta Mat. 63 (2010) 973-976. 
[23] C. Landron, E. Maire, J. Adrien, O. Bouaziz, M. Di Michiel, P. Cloetens, H. Suhonen, Resolution effect on the study of ductile damage using synchrotron x-ray tomography, Nucl. Inst. Meth. Phys. Res. B 284 (2012) $15-18$.

[24] J. Bontaz-Carion, Y. Pellegrini, X-ray microtomography analysis of dynamic damage in tantalum, Adv. Eng. Mat. 8 (6) (2006) 480-486.

[25] E. Maire, O. Bouaziz, M. Di Michiel, C. Verdu, Initiation and growth of damage in a dual-phase steel observed by x-ray microtomography, Acta Mat. 56 (18) (2008) 4954-4964.

[26] C. Landron, E. Maire, O. Bouaziz, J. Adrien, L. Lecarme, A. Bareggi, Validation of void growth models using x-ray microtomography characterization of damage in dual phase steels, Acta Mat. 59 (20) (2011) 7564-7573.

${ }_{520}$ [27] C. Martin, C. Josserond, L. Salvo, J. Blandin, P. Cloetens, E. Boller, Characterisation by x-ray micro-tomography of cavity coalescence during superplastic deformation, Scripta Mat. 42 (4) (2000) 375-381.

[28] L. Babout, E. Maire, J. Buffière, R. Fougères, Characterisation by X-Ray computed tomography of decohesion, porosity growth and coalescence in model metal matrix composites, Acta Mater. 49 (11) (2001) 2055-2063.

[29] A. Weck, D. S. Wilkinson, E. Maire, H. Toda, Visualization by x-ray tomography of void growth and coalescence leading to fracture in model materials, Acta Mater. 56 (12) (2008) 2919-2928.

[30] M. Torki, C. Tekoglu, J.-B. Leblond, A. Benzerga, Theoretical and numerical analysis of void coalescence in porous ductile solids under arbitrary loadings, Int. J. Plast. 91 (2017) 160-181.

[31] T. Morgeneyer, J. Besson, H. Proudhon, M. Starink, I. Sinclair, Experimental and numerical analysis of toughness anisotropy in AA2139 Al-alloy sheet, Acta Mat. 57 (2009) 3902-3915. 
32] S. Tang, A. M. Kopacz, S. Chan O’Keeffe, G. B. Olson, W. K. Liu, Three-dimensional ductile fracture analysis with a hybrid multiresolution approach and microtomography, J. Mech. Phys. Solids 61 (11) (2013) 21082124.

[33] M. Shakoor, A. Buljac, J. Neggers, F. Hild, T. Morgeneyer, L. Helfen, M. Bernacki, P.-O. Bouchard, On the choice of boundary conditions for micromechanical simulations based on 3D imaging, Int. J. Solids Struct. 112 (2017) 83-96.

[34] F. Fritzen, S. Forest, T. Boehlke, D. Kondo, T. Kanit, Computational homogenization of elasto-plastic porous metals, Int. J. Plasticity 29 (2012)

[35] Y. Zhang, C. Bajaj, B.-S. Sohn, 3D Finite Element Meshing from Imaging Data, Comput. Meth. Appl. Mech. Eng. 194 (48-49) (2005) 5083-5106.

[36] P. Young, T. Beresford-West, S. Coward, B. Notarberardino, B. Walker, A. Abdul-Aziz, An efficient approach to converting three-dimensional image data into highly accurate computational models., Phil. Trans. A 366 (1878) (2008) 3155-73.

[37] S. Osher, J. Sethian, Fronts propagating with curvature-dependent speed: Algorithms based on Hamilton-Jacobi formulations, J. Comput. Phys. 79 (1) (1988) 12-49.

[38] N. Sukumar, D. Chopp, N. Moës, T. Belytschko, Modeling holes and inclusions by level sets in the extended finite-element method, Comput. Meth. Appl. Mech. Eng. 190 (46-47) (2001) 6183-6200.

[39] E. Roux, M. Bernacki, P.-O. Bouchard, A level-set and anisotropic adaptive remeshing strategy for the modeling of void growth under large plastic 560 strain, Comput. Mat. Sci. 68 (32-46).

[40] D.-L. Quan, T. Toulorge, E. Marchandise, J.-F. Remacle, G. Bricteux, Anisotropic mesh adaptation with optimal convergence for finite elements 
using embedded geometries, Comput. Meth. Appl. Mech. Eng. 268 (2014) 65-81.

[41] M. Shakoor, B. Scholtes, P.-O. Bouchard, M. Bernacki, An efficient and parallel level set reinitialization method - Application to micromechanics and microstructural evolutions, Appl. Math. Modell. 39 (23-24) (2015) 72917302.

[42] J. Buffière, E. Maire, P. Cloetens, G. Lormand, R. Fougères, Characterisation of internal damage in a MMCp using X-ray synchrotron phase contrast microtomography, Acta Mater. 47 (5) (1999) 1613-1625.

[43] J. Buffière, E. Maire, J. Adrien, J. Masse, E. Boller, In Situ Experiments with X ray Tomography: an Attractive Tool for Experimental Mechanics, Exp. Mech. 50 (3) (2010) 289-305.

[44] B. Bay, T. Smith, D. Fyhrie, M. Saad, Digital volume correlation: threedimensional strain mapping using X-ray tomography, Exp. Mech. 39 (1999) $217-226$.

[45] T. Smith, B. Bay, M. Rashid, Digital volume correlation including rotational degrees of freedom during minimization, Exp. Mech. 42 (3) (2002) $272-278$.

[46] M. Bornert, J. Chaix, P. Doumalin, J. Dupré, T. Fournel, D. Jeulin, E. Maire, M. Moreaud, H. Moulinec, Mesure tridimensionnelle de champs cinématiques par imagerie volumique pour l'analyse des matériaux et des structures, Inst. Mes. Métrol. 4 (2004) 43-88.

[47] E. Verhulp, B. van Rietbergen, R. Huiskes, A three-dimensional digital image correlation technique for strain measurements in microstructures, J. Biomech. 37 (9) (2004) 1313-1320.

[48] S. Roux, F. Hild, P. Viot, D. Bernard, Three dimensional image correlation from X-Ray computed tomography of solid foam, Comp. Part A 39 (8) (2008) 1253-1265. 
[49] J. Rannou, N. Limodin, J. Réthoré, A. Gravouil, W. Ludwig, M. Baïetto, J. Buffière, A. Combescure, F. Hild, S. Roux, Three dimensional experimental and numerical multiscale analysis of a fatigue crack, Comp. Meth. Appl. Mech. Eng. 199 (2010) 1307-1325.

[50] A. Bouterf, J. Adrien, E. Maire, X. Brajer, F. Hild, S. Roux, Identification of the crushing behavior of brittle foam: From indentation to oedometric tests, J. Mech. Phys. Solids 98 (2017) 181-200.

[51] A. Buljac, M. Shakoor, M. Bernacki, P.-O. Bouchard, T. Morgeneyer, F. Hild, Numerical Validation Framework for Micromechanical Simulations based on Synchrotron 3D Imaging, Comput. Mech. 59 (3) (2017) 419-441.

[52] F. Hild, A. Bouterf, L. Chamoin, F. Mathieu, J. Neggers, F. Pled, Z. Tomičević, S. Roux, Toward 4D Mechanical Correlation, Adv. Mech. Simul. Eng. Sci. 3 (1) (2016) 1-26.

[53] M. Dong, C. Prioul, D. François, Damage effect on the fracture toughness of nodular cast iron: Part I. Damage characterization and plastic flow stress modeling, Metall. Mat. Trans. A 28 (11) (1997) 2245-2254.

[54] K. Zhang, J. Bai, D. François, Ductile fracture of materials with high void volume fraction, Int. J. Solids Struct. 36 (23) (1999) 3407-3425.

[55] N. Bonora, A. Ruggiero, Micromechanical modeling of ductile cast iron incorporating damage. Part I: Ferritic ductile cast iron, Int. J. Solids Struct. 42 (5-6) (2005) 1401-1424.

[56] G. Hütter, L. Zybell, M. Kuna, Micromechanisms of fracture in nodular cast iron: From experimental findings towards modeling strategies - A review, Eng. Fract. Mech. 144 (2015) 118-141.

[57] Z. Tomičević, J. Kodvanj, F. Hild, Characterization of the nonlinear behavior of nodular graphite cast iron via inverse identification. Analysis of uniaxial tests, Europ. J. Mech. A/Solids 59 (2016) 140-154. 
[58] H. Leclerc, J. Périé, S. Roux, F. Hild, Voxel-scale digital volume correlation, Exp. Mech. 51 (4) (2011) 479-490.

[59] H. Leclerc, J. Périé, F. Hild, S. Roux, Digital volume correlation: What are the limits to the spatial resolution?, Mech. \& Indust. 13 (2012) 361-371.

[60] A. Bouterf, S. Roux, F. Hild, J. Adrien, E. Maire, Digital volume correlation applied to X-ray tomography images from spherical indentation tests on lightweight gypsum, Strain 50 (5) (2014) 444-453.

[61] A. Buljac, T. Taillandier-Thomas, L. Helfen, T. Morgeneyer, F. Hild, Evaluation of Measurement Uncertainties of DVC applied to Laminography Data, J. Strain Analysis 53 (2) (2018) 49-65.

[62] N. Limodin, J. Réthoré, J. Adrien, J. Buffière, F. Hild, S. Roux, Analysis and artifact correction for volume correlation measurements using tomographic images from a laboratory X-ray source, Exp. Mech. 51 (6) (2011) 959-970.

[63] E. Roux, M. Shakoor, M. Bernacki, P.-O. Bouchard, A new finite element approach for modelling ductile damage void nucleation and growth analysis of loading path effect on damage mechanisms, Model. Simul. Mat. Sci. Eng. 22 (7) (2014) 075001.

[64] M. Shakoor, M. Bernacki, P.-O. Bouchard, A new body-fitted immersed volume method for the modeling of ductile fracture at the microscale: Analysis of void clusters and stress state effects on coalescence, Eng. Fract. Mech. 147 (2015) 398-417.

[65] M. Shakoor, P.-O. Bouchard, M. Bernacki, An adaptive Level-Set Method with enhanced volume conservation for simulations in multiphase domains, Int. J. Num. Meth. Eng. 109 (4) (2017) 555-576.

[66] D. Boffi, F. Brezzi, L. Demkowicz, R. Durán, R. Falk, M. Fortin, Mixed Finite Elements, Compatibility Conditions, and Applications, Vol. 1939 of Lecture Notes in Mathematics, Springer, Berlin, Heidelberg, 2008. 
[67] R. H. Wagoner, J. L. Chenot, Metal Forming Analysis, Cambridge University Press, 2001.

[68] P. Ludwik, Elemente der technologischen Mechanik, Verlag Von Julius Springer, Leipzig (Germany), 1909.

[69] A. Tarantola, Inverse Problems Theory. Methods for Data Fitting and Model Parameter Estimation, Elsevier Applied Science, Southampton (UK), 1987.

[70] J. Mottershead, M. Link, M. Friswell, The sensitivity method in finite element model updating: A tutorial, Mech. Syst. \& Signal Proc. 25 (7) (2011) 2275-2296.

[71] J. Collins, G. Hart, T. Hasselman, B. Kennedy, Statistical identification of structures, AIAA J. 12 (2) (1974) 185-190.

[72] E. Pagnacco, A. Caro-Bretelle, P. Ienny, Parameter Identification from Mechanical Field Measurements using Finite Element Model Updating Strategies, ISTE / Wiley, London (UK), 2012, pp. 247-274.

[73] H. Leclerc, J. Neggers, F. Mathieu, F. Hild, S. Roux, Correli 3.0, IDDN.FR.001.520008.000.S.P.2015.000.31500, Agence pour la Protection des Programmes, Paris (France) (2015). 